\title{
Inflammation, cardiovascular disease and cancer: moving toward predictive medicine
}

\author{
Paul M. Ridker MD MPH
}

— Cite as: CMAJ 2017 March 13;189:E382-3. doi: 10.1503/cmaj.161033

See related article at www.cmaj.ca/lookup/doi/10.1503/cmaj.160313

$n$ $\mathrm{n}$ this issue, Singh-Manoux and colleagues ${ }^{1}$ report that measures of low-grade systemic inflammation (e.g., C-reactive protein [CRP] and interleukin-6 [IL-6]) in midlife were strong independent predictors of all-cause, cardiovascular and cancerrelated mortality in the Whitehall II study cohort. These findings support evidence from more than a dozen prior prospective cohort studies with regard to CRP and all-cause mortality ${ }^{2}$ and more than 50 prior cohort studies showing that CRP and IL-6 predict future myocardial infarction and stroke. ${ }^{3,4}$ Because CRP and IL-6 are strongly correlated (both reflect upstream activation from interleukin-1), ${ }^{5}$ there is no clinical need to measure both factors, and of the two, CRP measured with a high-sensitivity assay (hs-CRP) is less expensive and has regulatory approval for clinical use. Following the multi-national JUPITER trial (Justification for the Use of Statins in Prevention: an Intervention Trial Evaluating Rosuvastatin), which showed large reductions in relative risk for first-ever cardiovascular events (myocardial infarction, stroke and cardiovascular death) in the rosuvastatin group among participants with low levels of cholesterol but elevated hs-CRP, the 2009 Canadian Cardiovascular Society guideline endorsed hs-CRP screening for cardiovascular risk prediction, particularly among patients at intermediate risk. ${ }^{6}$

Surprisingly, the third inflammatory biomarker in the study by Singh-Manoux and colleagues $-\alpha_{1}$-acid glycoprotein (AGP) - did not fare as well as either CRP or IL-6. This is important because the authors' motivation to perform the new analyses using the Whitehall II study cohort was to confirm or reject data from a recent metabolomics study that found AGP to be the strongest predictor of mortality in a nuclear magnetic resonance spectroscopy evaluation of 106 candidate biomarkers. ${ }^{7}$ That study, which used metabolomic discovery data from the Estonian Biobank and validated important biomarkers in a population-based cohort from Finland, suggested that four biomarkers predicted all-cause mortality: AGP, albumin, very-low-density lipoprotein particle size and citrate. However, because concomitant measures of CRP and IL- 6 were not done in the Estonian and Finnish cohorts, clinical comparisons could not be made. These kind of data are crucial because AGP and albumin both correlate with systemic low-grade inflammation and thus in turn with IL-6 and CRP.

\section{KEY POINTS}

- Metabolomic and proteomic studies are furthering the understanding of "predictive medicine."

- Inflammation is a biologic determinant of both cardiovascular disease and cancer and can be identified by measuring highsensitivity C-reactive protein or interleukin-6.

- Ongoing clinical trials will determine whether reducing inflammation reduces vascular event rates; if so, the clinical community will need to modify current concepts of residual risk to encompass residual cholesterol risk as well as the emerging concept of residual inflammatory risk.

In this context, Singh-Manoux and colleagues report that all three inflammatory biomarkers were associated with all-cause as well as cardiovascular and cancer-related mortality, both in univariate analyses and in analyses controlling for traditional risk factors. Overall, these effects weakened over time, with stronger associations in the first five years than in longer follow-up periods. However, when the authors controlled for all covariates and biomarkers simultaneously, AGP was no longer predictive. By contrast, the magnitude of effects for IL- 6 and CRP were largely similar for both cancer-related and cardiovascular mortality in the fully adjusted models (with small remaining differences between the two likely reflecting intercorrelations between IL-6 and CRP).

The analysis from Singh-Manoux and colleagues is thus an important reminder that data from metabolomics studies need to ensure that appropriate comparisons are made to established risk markers. Indeed, the metabolomics field has suffered from low levels of external validation. In this regard, it is worth comparing the earlier Estonian Biobank data with those reported by Cheng and colleagues $^{8}$ from the Offspring Cohort of the Framingham Heart Study, where higher concentrations of isocitrate, an intermediate of the citric acid cycle, were associated with lower odds of longevity.

What are the clinical implications of the current data? Measure of inflammation as a tool for cancer screening has found limited clinical utility, although immune-modulating therapies for cancer are a major new form of treatment. By contrast, since 
2000, it has been clear that measure of inflammation can be effective for cardiovascular screening, and more recently for the allocation of statin therapy. In fact, risk prediction algorithms for primary prevention that integrate data on inflammation (e.g., the Reynolds Risk Score) consistently outperform traditional risk prediction scores such as the Framingham Risk Score and the Pooled Cohort Equations from the American Heart Association and American College of Cardiology. ${ }^{9}$

It is unusual for biomarker development programs to result in a tool useful for risk prediction. However, biomarker discovery is crucial for thinking about new treatment targets. With regard to AGP, CRP and IL-6, what remains uncertain is whether reducing inflammation can reduce cardiovascular event rates. This important issue has been taken up by several investigative groups worldwide, and major hard-outcome trials are under way using agents such as low-dose methotrexate and colchicine (which are commonly used to treat rheumatoid arthritis and gout, respectively) as well as novel targeted agents such as canakinumab (a human monoclonal antibody that targets interleukin-1 $\beta$ ).

If the results of these trials are positive, then the clinical community will need to modify current concepts of residual risk to encompass not only residual cholesterol risk but also the emerging concept of residual inflammatory risk. ${ }^{10}$ This distinction leads to different therapies being used for different patients and represents an important move toward personalized cardiovascular medicine.

\section{References}

1. Singh-Manoux A, Shipley MJ, Bell JA, et al. Association between inflammatory biomarkers and all-cause, cardiovascular and cancer-related mortality. CMAJ 2017;189:E384-90.
2. Ridker PM. High sensitivity C-reactive protein as a predictor of all-cause mortality: implications for research and patient care. Clin Chem 2008;54:234-7.

3. Emerging Risk Factors Collaboration; Kaptoge S, Di Angelantonio E, Lowe G, et al. C-reactive protein concentration and risk of coronary heart disease, stroke, and mortality: an individual participant meta-analysis. Lancet 2010;375:132-40.

4. Kaptoge S, Seshasai SR, Gao P, et al. Inflammatory cytokines and risk of coronary heart disease: new prospective study and updated meta-analysis. Eur Heart J 2014;35:578-89.

5. Ridker PM. From C-reactive protein to interleukin-6 to interleukin-1: moving upstream to identify novel targets for atheroprotection. Circ Res 2016;118: 145-56.

6. Genest J, McPherson R, Frohlich J, et al. 2009 Canadian Cardiovascular Society/ Canadian guidelines for the diagnosis and treatment of dyslipidemia and prevention of cardiovascular disease in the adult -2009 recommendations. Can J Cardiol 2009;25:567-79.

7. Fischer K, Kettunen J, Würtz P, et al. Biomarker profiling by nuclear magnetic resonance spectroscopy for the prediction of all-cause mortality: an observational study of 17345 persons. PLoS Med 2014;11:e1001606.

8. Cheng S, Larson MG, McCabe EL, et al. Distinct metabolomic signatures are associated with longevity in humans. Nat Commun 2015;6:6791.

9. DeFilippis AP, Young R, Carrubba CJ, et al. An analysis of calibration and discrimination among multiple cardiovascular risk scores in a modern multiethnic cohort. Ann Intern Med 2015;162:266-75.

10. Ridker PM. Residual inflammatory risk: addressing the obverse side of the atherosclerosis prevention coin. Eur Heart J 2016;37:1720-2.

Competing interests: Paul Ridker is listed as a co-inventor on patents held by the Brigham and Women's Hospital that relate to the use of inflammatory biomarkers in cardiovascular disease and diabetes that have been licensed to Seimens and AstraZeneca. He has received research grants from the US National Heart, Lung, and Blood Institute and from Amgen, Novartis, AstraZeneca, Pfizer and Kowa.

This article was solicited and has not been peer reviewed.

Affiliation: Center for Cardiovascular Disease Prevention, Brigham and Women's Hospital, Harvard Medical School, Boston, Mass.

Correspondence to: Paul Ridker, pridker@partners.org 